\title{
Early discharge after major gynaecological surgery: advantages of fast track surgery
}

\author{
Jonathan Carter $^{1,2}$, Shannon Philp ${ }^{1,2}$, Vivek Arora ${ }^{1}$ \\ ${ }^{1}$ Sydney Gynaecological Oncology Group, Sydney Cancer Centre, Royal Prince Alfred Hospital, Sydney, Australia \\ ${ }^{2}$ The University of Sydney, Sydney, Australia \\ Email: jocarter@mail.usyd.edu.au
}

Received 20 February 2011; revised 21 March 2011; accepted 28 March 2011.

\begin{abstract}
Introduction: Fast Track Surgery (FTS) programs have been adopted by many specialties with documented improved patient outcomes and reduced length of stay (LOS). Methods: We initiated a FTS program in January 2008 and present our experience up to and including November 2010 on patients whose LOS was 2 days. Results: During the study period 242 patients had a laparotomy performed. Overall 54 (22.3\%) patients were discharged on day 2 . In the first year of initiating our FTS program $10 \%$ were discharged on day $2,25 \%$ in year 2 and $31 \%$ in year 3 . Twenty-two patients $(41 \%)$ had malignant pathology and of these, $16(73 \%)$ had local or regional spread and $6(27 \%)$ had distant spread. Forty patients $(\mathbf{7 4 \% )}$ had vertical midline incisions (VMI) performed. Surgery was classified as complex in $\mathbf{4 0}$ cases $(74 \%)$ and $6(11 \%)$ patients underwent staging lymph node dissection. Average patient BMI was 26.1 with $44 \%$ of patients considered overweight or obese. There were no intraoperative complications recorded. When compared to 188 patients whose LOS was greater than 2 days, the early discharge cohort were more likely to have benign pathology, more likely to be younger, to have a transverse incision, to have received COX II inhibitors, to have a lower net haemoglobin $(\mathrm{Hb})$ change and to tolerate early oral feeding. Conclusions: Increased clinical experience with FTS enables over $31 \%$ patients undergoing laparotomy to be safely discharged on day 2 without an increase in the readmission rate or morbidity.
\end{abstract}

Keywords: Fast Track Surgery; Gynaecology; Oncology

\section{INTRODUCTION}

In contemporary surgical care patients would often be admitted to hospital the day prior to planned surgery, undergo preoperative mechanical and antibiotic bowel preparation and have IV fluids running to keep them in fluid balance, prior to any surgical or anaesthetic insult. Intraoperatively patients were often volume loaded to maintain a filling pressure, had nasogastric tubes inserted, as well as pelvic drains to prevent development of collections, then spent 2 - 3 days nil by mouth (NBM) until bowel sounds were heard before being commenced on a graduated diet of clear liquids, free fluids, light diet and finally commenced on a regular diet 5 - 7 days post surgery. Patients were then discharged, on average 5 - 7 days post surgery [1].

Fast Track Surgery (FTS) or Enhanced Surgical Recovery (ESR) programs have been developed and refined in many specialties with documented improved patient outcomes and as a consequence earlier discharge form hospital and reduced length of stay (LOS) [1-5].

The aim of this study is to identify patients following a FTS program [6] who have been discharged earlier than anticipated, being day 2 post op after major gynaecological/gynaecological oncologic surgery and analyse elements that may have aided in their early discharge.

\section{METHODS}

After Ethics approval was granted the FTS Database was searched to identify patients operated upon by laparotomy between January 2008 and November 2010 and whose LOS was 2 days. Data was collected in a real time fashion and analysis undertaken in a retrospective fashion.

Our FTS protocol commences with preoperative patient counseling regarding the program. Patients are made aware that their anticipated LOS is 3 days, that narcotic analgesia will be limited and adequate analgesia provided by a combination of intraoperative paracoxib +/- transverse abdominis plane (TAP) block [7]. Postoperatively meloxicam is prescribed for 3 days with regular paracetamol. Early oral feeding by way of oral liquids are allowed on the night of surgery and patients are commenced on a light diet on post op day 1 with rapid progression thereafter. If significant abdominal 
distension or vomiting develops oral feeding is ceased. Movicol or Coloxyl with Senna is commenced routinely on post op day 1 and continued post discharge. All patients received perioperative Clexane 20 mg - 40 mg SCI which is continued until discharge. Intraoperatively mechanical sequential compression devices are employed and all patients have knee high TED stockings fitted preoperatively and are worn postoperatively for at least 1 month. Patients are mobilized on day 1 post surgery and catheters and IV fluids are removed on day 1 whenever possible. Patients are given an incentive spirometer and encouraged to use the device 6 times per hour. Criteria for discharge include the patient 1 ) adequately mobilizing without assistance, 2) tolerating early oral feeding, 3) having pain and discomfort controlled by oral analgesia and 4) having adequate home supervision after discharge. Post discharge patients receive a follow up phone call from our Clinical Nurse Consultant (CNC) within 3 days of discharge.

Data collected relate to 1) patient characteristics, 2) hospitalisation and 3) post-hospitalization. The patient characteristics collected were: age, weight, height, body mass index (BMI), medical insurance status and performance status. Hospitalization details included the procedure performed, type of incision (transverse or midline), operating time, complexity of surgery (simple vs. complex), intraoperative estimated blood loss, whether a transfusion was required, the preoperative $\mathrm{Hb}$, post operative $\mathrm{Hb}$ and the $\mathrm{Hb}$ change, whether the patient successfully completed early oral feeding and if the patient received COX II inhibitors. All inpatient complications were collected, including modified Royal Australian and New Zealand College of Obstetricians and Gynaecologists (RANZCOG) Quality Indicators. LOS was calculated by the difference between date of discharge and date of surgery. Post hospitalisation admissions and complications were also recorded.

Simple surgery was defined as benign low risk adnexal surgery or simple type 1 hysterectomy where formal ureteric dissection was not performed. All surgeries where formal pelvic sidewall dissection was undertaken were classified as "complex". Transverse incisions were classified according to the incision in the skin, irrespective of whether it was of Maylard type or Pfannenstiel.

Pathologically patients were classified on final pathological determination as either "benign" or "malignant". Patients with proliferating or borderline ovarian tumours were classified as "benign". Patients with malignant pathology were routinely reviewed 2 weeks postoperatively and then regularly thereafter, whilst those patients with benign pathology were reviewed $2-4$ weeks post operation.

According to International Standards, a BMI of 25 -
29.9 was considered overweight and $>30$ obese. Statistical analysis including descriptive statistics, t-test and ANOVA for nominal variables and chi-squared test for categorical data.

\section{RESULTS}

During the study period 242 patients had a laparotomy performed by the author (JC). Fifty-four (22.3\%) patients overall were discharged on day 2 . In year 1 after initiating the program $10 \%$ were discharged on day 2 , $25 \%$ in year 2 and 31\% in year 3 (Table 1).

Of those discharged on postop day 2, their average age was 47.6 years (Range: 20 - 74), with 25 (46\%) patients older than 50 years. Twenty two patients (41\%) had malignant pathology and of these 14 (25.9\%) had FIGO stage I disease, 2 (3.7\%) had FIGO stage II disease, 4 (7.4\%) had FIGO stage III disease and 2 (3.7\%) patient had FIGO stage IV disease. Sixteen (73\%) had local or regional spread and 6 (27\%) had distant spread.

Thirty one patients (57.4\%) had ovarian pathology, 15 (27.8\%) uterine pathology, 6 (11.1\%) cervical pathology and 2 patients (3.7\%) had other pathology. Thirty five patients (65\%) had private medical insurance and 40 patients (74\%) had VMI performed.

The mean operating time was 1.91 hours (Range: 0.92 hours - 3.3 hours). Two surgeries (4\%) lasted less than 1 hour, 33 lasted less than 2 hours (61\%) and 18 surgeries (33\%) lasted less than 3 hours and 1 surgery less than 4 hours.

Surgery was classified as complex in 40 cases (74\%) and $6(11 \%)$ patients underwent staging lymph node dissection.

Average patient weight was $68.95 \mathrm{~kg}$ with mean BMI 26.1 (range 17.5 - 44.5). Thirty patients (56\%) were classified as normal BMI, 11 (20\%) as overweight and 13 (24\%) as obese.

There were no blood transfusions and mean EBL at surgery was $196 \mathrm{~mL}$ (Range: $10 \mathrm{~mL}-900 \mathrm{~mL}$ ) and the mean $\mathrm{Hb}$ change was $8.3 \mathrm{~g} / \mathrm{L}$.

All patients were successfully fast tracked and tolerated early oral feeding, 51 (94\%) prescribed COX II inhibitors and 50 (93\%) had a "0" performance status described as "fully active". Four patients (7\%) had a performance status of " 1 ", described as "light restrictions".

There were no intraoperative complications recorded. One patient developed a wound infection and was readmitted 2 weeks post op. Two patients were transfused

Table 1. Incidence of discharge on day 2 post laparotomy compared with years after initiation of FTS.

\begin{tabular}{cccc}
\hline & Year 1 & Year 2 & Year 3 \\
\hline D/C Day 2 & 7/73 (10\%) & 25/99 (25.3\%) & 22/70 (31.4\%) \\
\hline
\end{tabular}


both had benign disease and severe menorrhagia. One was transfused both pre and post op and the second just postoperatively. One patient developed femoral neuropraxia that settled prior to discharge and one patient was a laparoscopic conversion after failed laparoscopic hysterectomy. One patient was diagnosed post discharge with a symptomatic PE. She was a 74 yr old woman with a BMI of 38 with recurrent low grade uterine sarcoma with a large mass. She was admitted for attempted debulking but was found to have unresectable, fixed pelvic disease. An omental biopsy only was performed.

When compared to 188 patients whose LOS was greater than 2 days, the early discharge cohort were more likely to have benign pathology $(\mathrm{P}<0.002)$, to be younger $(\mathrm{P}<0.0001)$, under age $50(\mathrm{P}=0.007)$, have a transverse incision rather than VMI $(\mathrm{P}=0.0002)$, have simple surgery performed $(\mathrm{P}=0.01)$, shorter operating times $(\mathrm{P}=0.0001)$, receiving COX II inhibitors $(\mathrm{P}=$ $0.04)$, more likely to have a " 0 " performance status $(\mathrm{P}=$ $0.002)$, a lower net $\mathrm{Hb}$ change $(\mathrm{p}=0.01)$, and more likely to tolerate early oral feeding $(\mathrm{p}=0.02)$ (Table 2$)$. There was no difference in tumour site, medical insurance status, BMI, proportion of overweight or obese patients, intraoperative transfusions, EBL at surgery, or complication rate

\section{DISCUSSION}

The improvement in surgical outcomes demonstrated in FTS programs has allowed as a consequence a reduction in the hospital LOS. This study has demonstrated that with experience, 1 in 3 patients undergoing a laparotomy for gynaecological surgery can be discharged on day 2 post surgery, without an increased morbidity or readmission rate $[8,9]$.

Whilst it is probably unrealistic to expect the LOS after laparotomy to be further reduced from 2 days, it is realistic to expect that with further refinements and enhancements to FTS programs a greater proportion of patients can expect a safe discharge on day 2 . With increasing experience of our team, and the appointment of a dedicated Fast Track Clinical Nurse Consultant (CNC), we have been able to increase the percentage of patients discharged on day 2 from $10 \%$ in the first year of the program to $25 \%$ in the second year and $31 \%$ in the third year after initiating a FTS program.

These improvements do not appear to be restricted to simple surgical cases in thin women, who have had transverse incisions and who lack private medical insurance. To the contrary, our data shows that 24 (44\%) patients discharged on day 2 were considered overweight or obese, 40 (74\%) had complex procedures performed, $35(65 \%)$ had private medical insurance and 40 (74\%) had vertical midline incisions.
Table 2. Comparison of patients discharged on day 2 with those discharged after day 2 .

\begin{tabular}{|c|c|c|c|}
\hline & $\begin{array}{l}\text { Discharge } \\
=\text { Day } 2 \\
(\mathrm{~N}=54)\end{array}$ & $\begin{array}{c}\text { Discharge } \\
>\text { Day } 2 \\
(\mathrm{~N}=188)\end{array}$ & Significance \\
\hline \multicolumn{4}{|l|}{ Age } \\
\hline Mean & 47.6 & 56.1 & $\mathrm{P}<0.0001$ \\
\hline$<50$ & 29 (53.7\%) & 63 (33.5\%) & $\mathrm{P}<0.01$ \\
\hline \multicolumn{4}{|l|}{ Performance } \\
\hline Fully Active & 49 (92.5\%) & 133 (70.7\%) & $\mathrm{P}<0.001$ \\
\hline Pathology & & & $\mathrm{P}<0.002$ \\
\hline Benign & 32 (59.3\%) & 67 (67.7\%) & \\
\hline Malignant & 22 (40.7\%) & $121(64.4 \%)$ & \\
\hline \multicolumn{4}{|l|}{ Stage } \\
\hline Stage I & $14(25.9 \%)$ & 65 (34.6\%) & $\mathrm{P}=0.01$ \\
\hline Stage II & $2(3.7 \%)$ & $6(3.2 \%)$ & \\
\hline Stage III & $4(7.4 \%)$ & 42 (22.3\%) & \\
\hline Stage IV & $2(3.7 \%)$ & $8(4.3 \%)$ & \\
\hline \multicolumn{4}{|l|}{ Incision } \\
\hline Transverse & 14 (25.9\%) & 13 (6.9\%) & $\mathrm{P}<0.0001$ \\
\hline VMI & 40 (74.1\%) & 175 (93.1\%) & \\
\hline \multicolumn{4}{|c|}{ Operating Time } \\
\hline Mean & 1.91 & 2.43 & $\mathrm{P}<0.0001$ \\
\hline \multicolumn{4}{|l|}{ Complexity } \\
\hline Simple & 14 (25.9\%) & 23 (12.2\%) & $\mathrm{P}<0.01$ \\
\hline Complex & $40(74.1)$ & 165 (87.8\%) & \\
\hline \multicolumn{4}{|l|}{ EBL } \\
\hline Hb Change & $8.3 \mathrm{~g} / \mathrm{L}$ & 11.3 & $\mathrm{P}<0.04 *$ \\
\hline COX II & $51(94.4 \%)$ & 157 (83.5\%) & $\mathrm{P}=0.02$ \\
\hline Tolerate EOF & 54 (100\%) & 165 (87.8\%) & $P<0.006$ \\
\hline
\end{tabular}

*Assuming equal variances.

Whilst not following FTS protocols as defined by Kehlet et al [1], Chase and colleagues are to be commended in reporting the largest series of gynaecologic surgical patients treated by a standard clinical pathway. Clinical pathways encompass some but not all of the elements essential to a FTS program. With 880 patients operated upon over a 7-year period, they found younger age, lower BMI, lower EBL at surgery, a post operative diagnosis of benign ovarian neoplasm and non-radical dissection significant factors for early discharge [10]. In this series an incredible 322 patients (37\%) were able to 
be discharged on day 2, setting the benchmark for all others following the principals of FTS to aspire to. Our findings are similar to Chase et al, in that younger age, ovarian tumours, benign pathology, transverse skin incisions, decreased operating time, early oral feeding, use of COX II inhibitors and net haemoglobin change were all significantly different in the early discharge group when compared to the group of patients discharged after day 2. In addition, with our greater experience, we have found in our third year after adopting FTS principles, $31.4 \%$ of our patients can now be discharged on day 2 . We did not find insurance status, weight, BMI or estimated blood loss to be significantly different between the groups.

Both Chase et al and our own group have questioned the reported perceived benefits of laparoscopic surgery in light of results achieved with FTS laparotomy $[6,10]$. Recent evidence from the GOG LAP2 study support our position. This is the largest prospective randomized study in the world comparing laparoscopic surgery for endometrial cancer to non-fast tracked laparotomy patients. In summary there were similar rates of intra-operative complications, with a significant number of patients converted to laparotomy due to poor visibility, metastatic cancer or bleeding. Median LOS of non-fast tracked laparotomy patients was 4 days and 3 days for laparoscopy patients [11]. Furthermore, whilst quality of life (QOL) was enhanced across many parameters in the laparoscopy group at 6 weeks, these differences were not significant by 6 months. Whilst cross trial comparisons are statistically invalid, one can only speculate whether the 1 day difference in LOS would be negated if the laparotomy patients in this study were fast tracked [12] Similar results have also been published by Mouritis and colleagues [13] who have reported a median LOS of 5 days for their non-fast tracked hysterectomy patients compared to 2 days for laparoscopic hysterectomy patients and no difference in major complication rate.

Further enhancements to our FTS program which will allow a greater proportion of patients to be safely discharged on day 2 include enhancing preoperative consultations to further emphasise the rationale of FTS and encouraging patients to contribute by agreeing to discharge when medically appropriate. A number of our patients discharged on day 3 could have been discharged on day 2 had there been appropriate supervision at home on discharge. Improving GI function is also an area we have identified as worthy of further study. Simple techniques such as initiating a regular chewing gum regimen after surgery to stimulate bowel function has been shown to be effective and would add little financial impost and minimal risk of increased morbidity [14-19]. Health care organisations and Hospital Administrators also derive significant financial benefit from a FTS program and should provide funding for the initiation and maintenance of such programs.

This study has shown that with experience over 30\% patients undergoing laparotomy on a FTS program, can be safely discharged on day 2 without an increase in the readmission rate or morbidity.

\section{REFERENCES}

[1] Kehlet, H. and Wilmore, D.W. (2002) Multimodal strategies to improve surgical outcome. American Journal of Surgery, 183, 630-641. doi:10.1016/S0002-9610(02)00866-8

[2] Australian Safety and Efficacy Register of New Interventional Procedures-Surgical (ASERNIPS), The Royal Australasian College of Surgeons (2009) Brief review: Fast-track surgery and enhanced recovery after surgery (ERAS) programs.

[3] Fearon, K., Ljungqvist, O., Von Meyenfeldt, M., Revhaug, A., Dejong, C., Lassen, K., et al. (2005) Enhanced recovery after surgery: A consensus review of clinical care of patients undergoing colonic resection. Clinical Nutrition, 24, 466-477. doi:10.1016/j.clnu.2005.02.002

[4] Kehlet, H. and Wilmore, D.W. (2008) Evidence-based surgical care and the evolution of fast-track surgery. Annals of Surgery, 248, 189-198. doi:10.1097/SLA.0b013e31817f2c1a

[5] Pruthi, R., Niesen, M., Smith, A., Nix, J., Schultz, H. and Wallen, E. (2010) Fast track program in patients undergoing radical cystectomy: Results in 362 consecutive patients. Journal of the American College of Surgeons, 210, 93-99. doi:10.1016/j.jamcollsurg.2009.09.026

[6] Carter, J., Szabo, R., Sim, W., Pather, S., Philp, S., Nattress, K., et al. (2010) Fast track surgery in gynaecological oncology. A clinical audit. Australian and New Zealand Journal of Obstetrics and Gynaecology, 50, 159-163. doi:10.1111/j.1479-828X.2009.01134.X

[7] McDonnell, J.G., O'Donnell, B., Curley, G., Heffernan, A., Power, C. and Laffey, J.G. (2007) The analgesic efficacy of transversus abdominis plane block after abdominal surgery: A prospective randomized controlled trial. Anesthesia \& Analgesia, 104, 193-197.

[8] Marx, C., Rasmussen, T., Jakobsen, D.H., Ottosen, C., Lundvall, L., Ottesen, B., et al. (2006) The effect of accelerated rehabilitation on recovery after surgery for ovarian malignancy. Acta Obstetricia et Gynecologica Scandinavica, 85, 488-492. doi:10.1080/00016340500408325

[9] Massad, L., Vogler, G., Herzog, T. and Mutch, D. (1993) Correlates of length of stay in gynecologic oncology patients undergoing inpatient surgery. Gynecologic Oncology, 51, 214-218. doi:10.1006/gyno.1993.1275

[10] Chase, D.M., Lopez, S., Nguyen, C., Pugmire, G.A. and Monk, B.J. (2008) A clinical pathway for postoperative management and early patient discharge: Does it work in gynecologic oncology? American Journal of Obstetrics and Gynecology, 199, e1-e7.

[11] Walker, J.L., Piedmonte, M.R., Spirtos, N.M., Eisenkop, S.M., Schlaerth, J.B., Mannel, R.S., et al. (2009) Lapar- 
oscopy compared with laparotomy for comprehensive surgical staging of uterine cancer: Gynecologic oncology group study LAP2. Journal of Clinical Oncology, 27, 5331-5336.

[12] Kornblith, A.B., Huang, H.Q., Walker, J.L., Spirtos, N.M., Rotmensch, J. and Cella, D. (2009) Quality of life of patients with endometrial cancer undergoing laparoscopic international federation of gynecology and obstetrics staging compared with laparotomy: A gynecologic oncology group study. Journal of Clinical Oncology, 27, 5337-5342.

[13] Mourits, M,, Bijen, C., Art, H., ter Brugge, H., van der Sijde, R., Paulsen, et al. (2010) Safety of laparoscopy versus laparotomy in early-stage endometrial cancer: A randomised trial. Lancet Oncology, 16, 1-9.

[14] de Castro, S., van den Esschert, J., van Heek, N., Dalhuisen, S., Koelemay, M., Busch, O., et al. (2008) A systemic review of the efficacy of gum chewing for the amelioration of postoperative ileus. Digestive Surgery, 25, 39-45. doi:10.1159/000117822

[15] Edward, J., Fitzgerald, F. and Irfan, A. (2009) Systematic Review and Meta-Analysis of Chewing-Gum Therapy in the Reduction of Postoperative Paralytic Ileus Following Gastrointestinal Surgery. World Journal of Surgery, 33, 2557-2566.

[16] Hansen, C., Sorensen, M., Moller, C., Ottesen, B. and Kehlet, H. (2007) Effect of laxatives on gastrointestinal functional recovery in fast-track hysterectomy: A doubleblind, placebo-controlled randomized study. American Journal of Obstetrics and Gynecology, 196, 311e1$311 \mathrm{e} 7$.

[17] Ljungqvist, O. and Soreide, E. (2003) Preoperative fasting. British Journal of Surgery, 90, 400-406. doi:10.1002/bjs.4066

[18] Macmillan, S., Kammerer-Doak, D., Rogers, R. and Parker, K. (2000) Early feeding and the incidence of gastrointestinal symptoms after major gynecologic surgery. Obstetrics \& Gynecology, 96, 604-608. doi:10.1016/S0029-7844(00)00957-1

[19] Schilder, J., Hurteau, J., Look, K., Moore, D., Raff, G., Stehman, F., et al. (1997) A prospective controlled trial of early postoperative oral intake following major abdominal gynecologic surgery. Gynecologic Oncology, 67, 235-240. doi:10.1006/gyno.1997.4860 